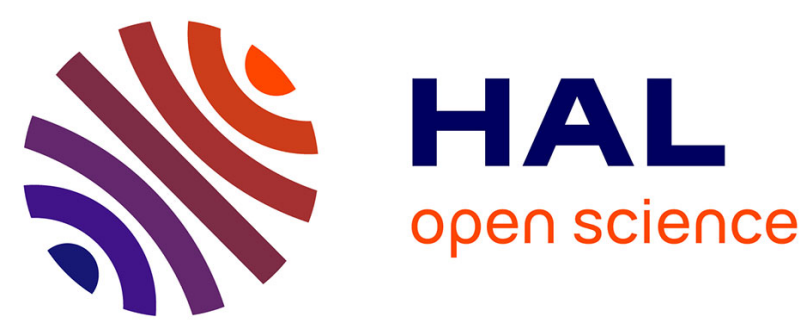

\title{
A pervasive multi-sensor data fusion for smart home healthcare monitoring
}

\author{
Hamid Medjahed, Dan Istrate, Jérôme Boudy, Jean-Louis Baldinger,
} Bernadette Dorizzi

\section{- To cite this version:}

Hamid Medjahed, Dan Istrate, Jérôme Boudy, Jean-Louis Baldinger, Bernadette Dorizzi. A pervasive multi-sensor data fusion for smart home healthcare monitoring. FUZZ 2011: IEEE International Conference on Fuzzy Systems, Jun 2011, Taipei, Taiwan. pp.1466 - 1473, 10.1109/FUZZY.2011.6007636 . hal-01302225

\section{HAL Id: hal-01302225 \\ https://hal.science/hal-01302225}

Submitted on 13 Apr 2016

HAL is a multi-disciplinary open access archive for the deposit and dissemination of scientific research documents, whether they are published or not. The documents may come from teaching and research institutions in France or abroad, or from public or private research centers.
L'archive ouverte pluridisciplinaire HAL, est destinée au dépôt et à la diffusion de documents scientifiques de niveau recherche, publiés ou non, émanant des établissements d'enseignement et de recherche français ou étrangers, des laboratoires publics ou privés. 


\section{A Pervasive Multi-sensors Data Fusion for Smart Home Healthcare Monitoring}

\author{
Hamid Medjahed \\ Dan Istrate \\ ESIGETEL, LRIT \\ Avon-Fontainebleau, France \\ Email: \{hamid.medjahed,dan.istrate\}@esigetel.fr
}

\author{
Jerome Boudy \\ Jean-Louis Baldinger \\ Bernadette Dorizzi \\ Telecom SudParis, EPH, Evry, France \\ Email: \{jerome.boudy,jean-louis.baldinger $\} @$ it-sudparis.eu
}

\begin{abstract}
Today elderly people are the fastest growing segment of the developing countries population, and they desire to live as independently as possible. But independent lifestyles come with risks and challenges. Medical in-home telemonitoring (and, more generally, telemedicine) is a solution to deal with these challenges and to ensure that elderly people can live safely and independently in their own homes for as long as possible. In this context we propose an automatic in-home healthcare monitoring system for several uses and to meet the needs identified above. The proposed telemonitoring system is a multimodal platform with several sensors that can be installed at home and enables us to have a full and tightly controlled universe of data sets. It integrates elderly physiological and behavioral data, the acoustical environment of the elderly, environmental conditions and medical knowledge. Each modality is processed and analyzed by specific algorithms. A data fusion approach based on fuzzy logic with a set of rules directed by medical recommendations, is used to fuse the various subsystem outputs. This multimodal fusion increases the reliability of the whole system by detecting several distress situations. In fact this fusion approach takes into account temporary sensor malfunction and increases the system reliability and the robustness in the case of environmental disturbances or material limits (Battery, RF range, etc.). The Fuzzy logic fusion methods brings high flexibility to the telemonitoring platform especially in combining modalities or adding other sensors. The proposed telemonitoring system will ensure pervasive in-home health monitoring for elderly people.
\end{abstract}

\section{INTRODUCTION}

France's population continues to age significantly and a statistic study carried out by the French national institute of statistic and economic studies (INSEE) shows a new distribution of age classes in France. In fact, almost one in three people will be over 60 years in 2050, against one in five in 2005, and France will have over 10 million of people over 75 years and over 4 million of people over 85 years. Therefore, the aging population and the increase in life expectancy have led to new models of aging where technology can play a role in monitoring the quality of life, by detecting or even predicting adverse events and hence supporting independence. Automatic in-home telemonitoring of distress situations has been a common focus in Gerontechnology [1] because medical telemonitoring at home, is an interesting solution compared to health facility institutions for the elderly, since it offers medical surveillance in a familiar atmosphere for the patient.

To address these issues, researchers are developing technologies to enhance a resident's safety and monitor health conditions using sensors and other devices. Numerous projects are carried out in the world especially in Europe, Asia and North America, on the home healthcare telemonitoring topic. They aim for example to define a generic architecture for such telemonitoring systems [2], to conduct experiment of a remote monitoring system on a specific category of patients [3] (Insufficient cardiac heart, asthma, diabets, patients with Alzheimer's disease, or cognitive impairement, etc.), or to build smart apartments [4], sensors and alarm systems adapted to the healthcare telemonitoring requirements [5].

However, little research exists to motivate and guide such technology. For example most monitoring systems use some form of learning method to discriminate between different types of normal and abnormal events. These algorithms require large amounts of training data that can be dificult to obtain, especially data describing abnormal events that are by definition scarce occurrences. The most crucial issue for all these systems is the lack of experimental data and information representing many situations and several person profiles. Most of these systems also take into account only one modality, like medical sensors (Blood pressure, pulse, oxymeter) or localization sensors (Infrared or contacts) to survey patient. But among established medical remote systems, there are few commercial solutions and business models.

In this paper we present a new multimodal system called EMUTEM (Environnement Multimodal pour la Télévigilance Médicale) for in home health care monitoring. The platform developed within this framework research manages a system consisting of:

- A set of microphones placed in all rooms of the elderly's house, that allow remote monitoring of the acoustical environment of the elderly (Anason [6]).

- A wearable device called RFpat[7] that can measure physiological data like ambulatory pulse heart rate, detect posture, fall of the equipped person and his activity rate.

- A set of infrared sensors that detect the person's presence in a given home part, his posture and also his movement (Gardien [7]).

- A set of domotic sensors like contact sensors, temperature sensors, smoke sensors and several other domotic sensors for environment conditions monitoring (Gardien [7]).

Subsystem data streams have been separately processed with 
suitable algorithms for abnormal situations detection. In order to maximize correct classification performance between normal and distress situations, data fusion over the different sensors types is studied. The area of data fusion has generated great interest among researchers in many science and engineering disciplines. We have identified two major classes of fusion techniques: (1) Those that are based on probabilistic models (such as Bayesian reasoning [8] and geometric decision reasoning), but their performance is limited when the data are too complex, therefore the model is uncontrollable. (2) Those based on connectionist models (such as neural networks MLP [9] and SVM [10]) which are very powerful because they can model the strong nonlinearity of data but with complex architecture, thus lack of intelligibility. Based on those facts and considering the complexity of the data to process (audio, physiologic and multisensory measurements) plus the difficulty of the statistical modeling of abnormal situation, fuzzy logic has been found useful to be the decision module of our multimodal monitoring system. Fuzzy logic, a mathematical formalism able to represent various types of uncertainty, provides tools with potential interest. Firstly, fuzzy logic can gather performance and intelligibility and it deals with imprecision and uncertainty. Secondly, fuzzy logic offers a wide range of operators for combining the output of different subsystems aimed at different goals. Finally the intrinsic ability of fuzzy logic to integrate numerical ('fuzzy') and symbolic ('logic') computation suggests its use as common formalism to help integrate numeric control and symbolic planning (academic knowledge).

The originality of this research is the combination of various modalities in the home, about its inhabitant and their surroundings. The new multimodal data fusion approach based on fuzzy logic allows high flexibility for the EMUTEM platform especially in combining modalities or adding other sensors. It constitutes an interesting benefit and impact for the elderly person sufering from loneliness. This work complements the stationary smart home environment in bringing to bear its capability for integrative continuous observation and detection of critical situations.

\section{The Telemonitoring System}

We define a smart environment as one with the ability to adapt the environment to the inhabitants and meet the goals of comfort and efficiency. In order to achieve these goals, our first aim was focused on providing such as environment. We consider our system as an intelligent agent, which perceives the state of the environment by using sensors and acts consequently with device controllers.

\section{A. Environement Sensing and Data Collection}

The experimental area is a surface of $20 \mathrm{~m} 2$ in our laboratory which is arranged in two rooms with a technical area in order to evaluate and to supervise experiments. The hardware framework is reported in Figure 1. It integrates smart sensors (Infrared, change state sensors, audio, physiological,) linked to a PC. Microphones for audio monitoring are linked to the PC

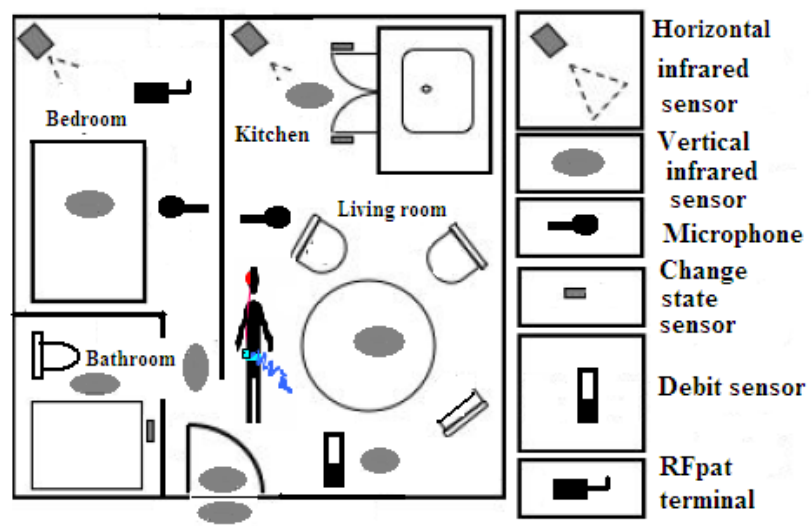

Fig. 1. In-home sensor disposal.

through an external sound card (in order to allow good signal to noise ratio independent from the PC), and can be interpreted as a single smart audio sensor achieved by Anason software. Infrared sensors are fixed on specific places of the house in walls and ceiling. They are in permanent communication via radio frequency communication, with one receiver, which is connected to the USB port of the PC. Change state sensors transmit also information to this receiver through radio frequency communication. The powerline is used to get back data from the receiver for software processing. The weareble device RFpat is caried by the elderly and continuously monitors his physiological data and emergency calls. It transmits these data to an indoor reception base station via ZigBee.

Figure 2 shows the software architecture of the multimodal platform EMUTEM. It provides a general user interface which encapsulates the Anason subsystem. It is implemented under LabWindows/CVI software and communicates with RFpat and Gardien sub-systems by a client-server model using TCP/IP and appropriate application protocols. Gardien is implemented in $\mathrm{C}++$ and recovers data every $500 \mathrm{~ms}$. RFpat is also implemented in $\mathrm{C}++$ and receives data from a receiver every $30 \mathrm{~s}$. The use of the inter-module communication through TCP/IP sockets allows each module (subsystem) to be run on

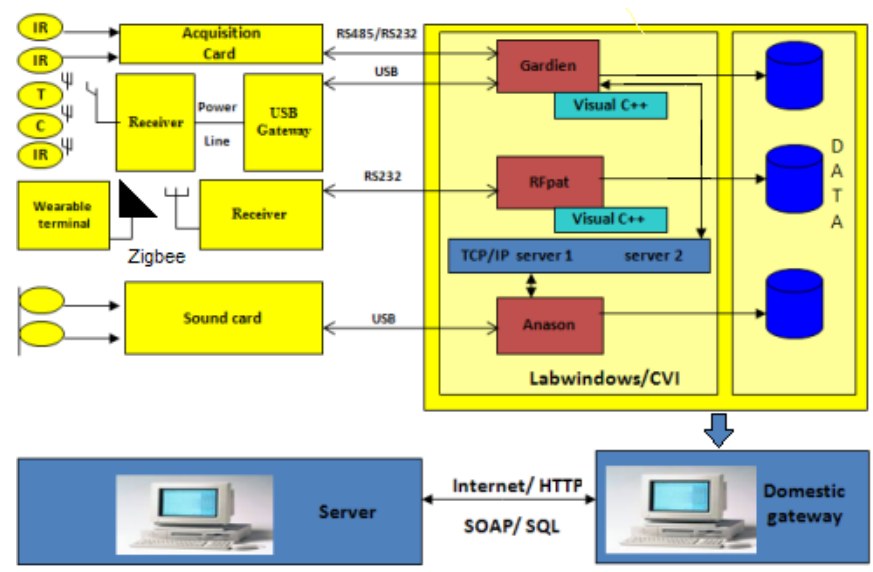

Fig. 2. EMUTEM software architecture. 
a different computer, and to synchronize each telemonitoring modality channel.

The user can interact with the system via internet navigator and supervises the different applications. This feedback provides a significant help to the system manipulation and the system flexibility obtained through TCP/IP sockets communication allows adding other potential sensors such as a heart monitoring sensors (ECG). Data acquired from sensors are stored in the local computer directly as text files assigned to each modality. Data also could be exchanged using http or ftp protocol via web services technologies SOAP, and saved in a dedicated server.

\section{Distress Situation Detection}

Detecting and gathering data about the elderly person and his environment is the first step and one of the most fundamental tasks in building intelligent telemonitoring systems. With the increasing intelligence expectation, using multiple sensors is the only way to obtain the required breadth of information, and fusing the outputs from these multiple sensors is often the only way to obtain the required depth of information when a single sensing modality is inadequate [13]. However, in our telemonitoring context different sensors use different physical principles, cover different information space, and generating data in different formats at different sampling rates. The obtained data have different resolution, accuracy, and reliability properties.

Based on those effects, the key to produce the required detection is to use the right method that properly fuses the provided data from various sources. This is what multimodal data fusion stands for. Therefore, typical multisensors data fusion methods are analyzed in this section, in seeking for a most generalizable and adaptable method.

\section{A. Adapted Approaches for Multi-sensors Data Fusion}

To select a suitable method for EMUTEM's multimodal data fusion module, we have probabilistic approaches whose performance can be reconsidered in our application for many reasons. The classical inference method quantitatively compares the probability that an observation can be attributed to a given assumed hypothesis. But it has the following major disadvantages [14], (1) difficulty in obtaining the density functions that describe observations used to classify the object, (2) complexities that arise when multivariate data are encountered, (3) its capability to assess only two hypotheses at a time, and (4) its inability to take direct advantage of a priori likelihood probabilities.

The Bayesian inference method [15] also has some weaknesses that prevent it from being used in our multimodal data fusion module. The key limits are: (1) difficulty in defining a priori probabilities, (2) complexities when there are multiple potential hypotheses and multiple conditionally dependent events, (3) mutual exclusivity required for competing hypotheses, and (4) inability to account for general uncertainty and to represent imprecision.

Even if Dempster-Shafer methods [16] use a general level of uncertainty, they cannot be the main data fusion method for two reasons: the difficulty to estimate mass function and their restrict domain of application.

The neural networks method [17] is not adapted to EMUTEM's Data fusion module because of drawbacks. First, the mapping mechanism is not well understood even if the network can provide the desired behavior, only in the simplest toy-like problems does examination of the weights in the trained network give any clue as to the underlying analytical connection between the inputs and outputs. Second, the neural network method is, generally speaking, not suitable to work in a dynamic sensor configuration environment, because each sensor needs a unique input node and each possible sensor-set configuration needs to be specifically trained. Third, the complex architecture of neural networks prevents expers adding their knowledge easily.

SVM methods [18], despite their transit in the characteristics space which is disconnected from any physical reality, could fulfill the requirement of intelligibility because only support vectors are important in identifying margins between classes. However, it is necessary that boundaries between classes are rendered intelligible by a graphical way in the space of inputs. This vision must take into account an input space of any size even if greater than 3. In this case, the SVM identifies a large majority of learning examples as support examples. It means that an analyst should remember too many relevant individuals for the construction of boundaries between classes and this is impossible.

The fuzzy logic method [19] is the proposed way to meet these challenges of this multimodal data fusion application. According to the nature of data to process in EMUTEM platform, fuzzy logic is the well adapted approach for the telemonitoring decision. It deals with inaccuracy and uncertainty. It allows a great flexibility to combine several sensors.

\section{B. Background About Fuzzy Logic}

The use of fuzzy logic in EMUTEM fusion is motivated by two main reasons from a global point of view:

- Firstly the characteristics of the data to merge which are measurements obtained from different sensors, thus they could be imprecise and imperfect. These data will be classified into two class: normal situations and distress ones, that are fuzzy because there is no clear limit between them.

- Secondly, the history of fuzzy logic shows that it is used in many steps which are necessary for a data classification application.

Rather than creating new methods of fusion and classification based on entirely different approaches, fuzzy logic fits naturally in the expression of the problem of classification, and tends to make a generalization of the classification methods that already exist. Taking into account the four steps of a recognition system proposed by Bezdek et Pal [21], fuzzy logic is very useful for these steps.

1) How To Do Fuzzy Logic: The main concept of fuzzy logic is that many problems in the real world are imprecise rather than exact. It is believed that the effectiveness of the 
human brain is not only from precise cognition, but also from fuzzy concepts, fuzzy judgment, and fuzzy reasoning. An advantage of fuzzy classification techniques lies in the fact that they provide a soft decision, a value that describes the degree to which a pattern fits within a class, rather than only a hard decision, i.e., a pattern matches a class or not. Fuzzy logic is based on natural language which makes it a quite attractive field in artificial intelligence. It allows the natural description of problem domains, in linguistic terms, rather than in terms of relationships between precise numerical values.

A fuzzy set, as the foundation of fuzzy logic, is a set without a hard, clearly sharp defined boundary. A fuzzy set extends a standard set by allowing degrees of membership of an element to this set, measured by real numbers in the $[0,1]$ interval. If $X$ is the universe of discourse (the input space variable) and its elements are denoted by $x$, then a fuzzy set $A$ on $X$ is defined as a set of ordered pairs $\left(x, \mu_{A}(x)\right)$ such that:

$$
A=\left\{x, \mu_{A}(x) \mid x \in X, 0 \leq \mu_{A}(x) \leq 1\right\}
$$

where $\mu_{A}(x)$ in equation 1 , is the membership function (MF) of each $x$ in $A$. In contrast to classical logic where the membership function $\mu_{A}(x)$ of an element $x$ belonging to a set $A$ could take only two values: $\mu_{A}(x)=0$ if $x \in A$ or $\mu_{A}(x)=1$ if $x \notin A$, fuzzy logic introduces the concept of membership degree of an element $x$ to a set $A$ and $\mu_{A}(x) \in$ $[0 ; 1]$, here we speak about the truth value.

A typical fuzzy logic inference system has four components: a fuzzification, a fuzzy rule base, an inference engine, and a defuzzification.

1) Fuzzification: The first step in fuzzy logic is to convert the measured data into a set of fuzzy variables. It is done by giving value (these will be our variables) to each of a membership function set. Membership functions take a different shape. A Triangular membership function with straight lines can formally be defined as follows:

$$
\Lambda(x, a, b, c)=\left\{\begin{aligned}
0, & x \leq a \\
(x-a) /(b-a), & a \leq x \leq b \\
(c-x) /(c-b), & b \leq x \leq c \\
0, & x \geq c
\end{aligned}\right.
$$

Trapezoidal Function Furnished in the equation 3.

$$
f(x, a, b, c)=\left\{\begin{aligned}
0, & x \leq a \\
(x-a) /(b-a), & a \leq x \leq b \\
1, & b \leq x \leq c \\
(d-x) /(d-c), & c \leq x \leq d \\
0, & x \geq d
\end{aligned}\right.
$$

A Gaussian membership function with the parameters $m$ and $\sigma$ to control the center and width of the membership function is defined by

$$
G(x, m, \sigma)=e^{\frac{-(x-m)^{2}}{2 \sigma^{2}}}
$$

The generalized Bell function depends on three parameters $a, b$, and $c$ is given by

$$
f(x, a, b, c)=\frac{1}{1+\|(x-c) / a\|^{2 b}}
$$

There are also other membership functions like sigmoidally shaped function, single function etc... The choice of the function shape is iteratively determined, according to the type of data and taking into account the experimental results.

2) Fuzzy rules and inference system: The fuzzy inference system uses fuzzy equivalents of logical AND, OR and NOT operations to build up fuzzy logic rules. An inference engine operates on rules that are structured in an IF-THEN format. The IF part of the rule is called the antecedent, while the THEN part of the rule is called the consequent. Rules are constructed from linguistic variables. These variables take on the fuzzy values or fuzzy terms that are represented as words and modeled as fuzzy subsets of an appropriate domain. There are several types of fuzzy rules, we only mention the two main rules used in our system:

- Mamdani rules [22] which are of the form: If $x_{1}$ is $A_{1}$ and $x_{2}$ is $A_{2}$ and...and $x_{p}$ is $A_{p}$ then $y_{1}$ is $C_{1}$ and $y_{2}$ is $C_{2}$ and...and $y_{p}$ is $C_{p}$. Where $A_{i}$ and $C_{i}$ are fuzzy sets that define the partition space. The conclusion of a Mamdani rule is a fuzzy set. It uses the algebraic product and the maximum as T-norm and S-norm respectively, but there are many variations by using other operators.

- Takagi/Sugeno rules [22]: If $x_{1}$ is $A_{1}$ and $x_{2}$ is $A_{2}$ and...and $x_{p}$ is $A_{p}$ Then $y=b_{0}+b_{1} x_{1}+$ $b_{2} x_{2}+\ldots+b_{p} x_{p}$. In the Sugeno model the conclusion is numerical. The rules aggregation is in fact the weighted sum of rules outputs.

3) Defuzzification: The last step of a fuzzy logic system consists in turning the fuzzy variables generated by the fuzzy logic rules into real values again which can then be used to perform some action. There are different defuzzification methods, in our platform decision module we could use Centroid Of Area (COA), Bisector Of Area (BOA), Mean Of Maximum (MOM), Smallest Of Maximum (SOM) and Largest Of Maximum (LOM). Equations 6, 7, 8 and 9 illustrate them.

$$
\begin{gathered}
Z_{C O A}=\frac{\sum_{i=1}^{n} \mu_{A}\left(x_{i}\right) x_{i}}{\sum_{i=1}^{n} \mu_{A}\left(x_{i}\right)} \\
Z_{B O A}=x_{M} ; \sum_{i=1}^{M} \mu_{A}\left(x_{i}\right)=\sum_{j=M+1}^{n} \mu_{A}\left(x_{j}\right) \\
Z_{M O M}=\frac{\sum_{i=1}^{N} x_{i}^{*}}{N} \\
Z_{S O M}=\min \left(x_{i}^{*}\right) \text { and } Z_{L O M}=\max \left(x_{i}^{*}\right)
\end{gathered}
$$

where $x_{i}^{*}(i=1,2, \ldots, N)$ reach the maximal values of $\mu_{A}\left(x_{i}\right)$.

$Z$ is the defuzzification value and $\mu_{A}\left(x_{i}\right)$ is the membership function. 


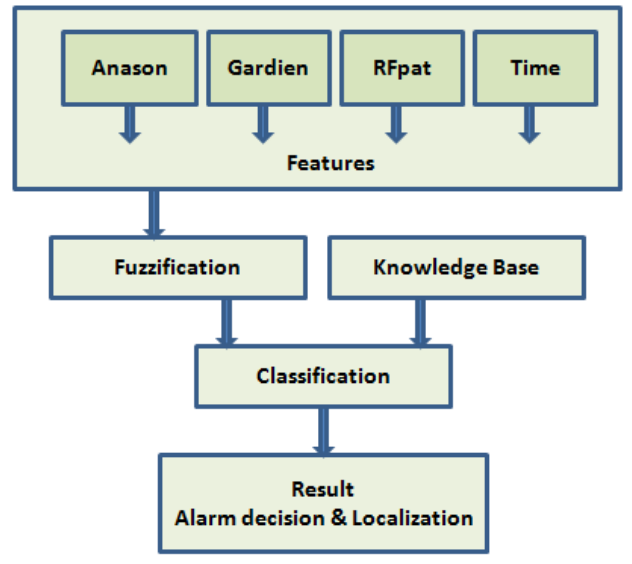

Fig. 3. Structure of the fuzzy classifier.

\section{IMPLEMENTATION}

\section{A. Fuzzy Classifier}

The main advantages of using fuzzy logic system are the simplicity of the approach and the capacity of dealing with the complex data acquired from the subsystems discribed previously in the second section. Fuzzy set theory offers a convenient way to do all possible combinations with these data. Fuzzy set theory is used in this system to determine the most likely distress situations that might occur for elderly persons in their home. The data fusion is carried out at three different levels: for sound/speech environement at the decision level, for smart home sensors system at the input data level and for the wearable physiological sensor at the representation level.

\section{B. Input and Output fuzzification}

The first step for implementing the fuzzy logic multimodal data fusion approach is the fuzzification of outputs and inputs of the fuzzy inference system (FIS) obtained from each subsystem.

From Anason subsystem three inputs are built. The first one is the sound environment classification. Sound classes are labeled on a numerical scale according to their alarm level.
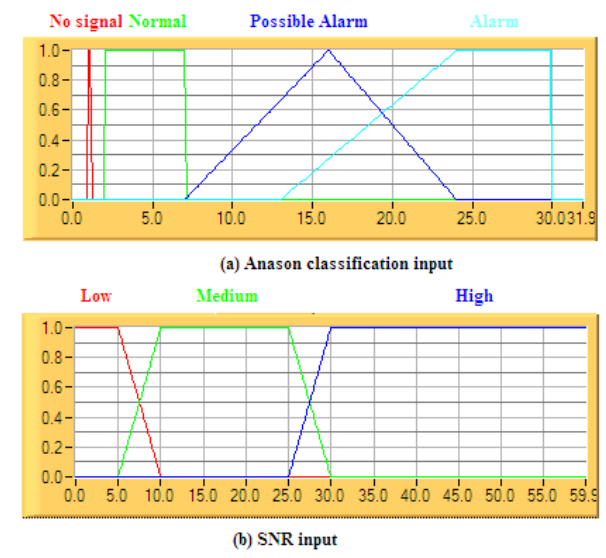

Fig. 4. Fuzzy sets defined for sound environment inputs.

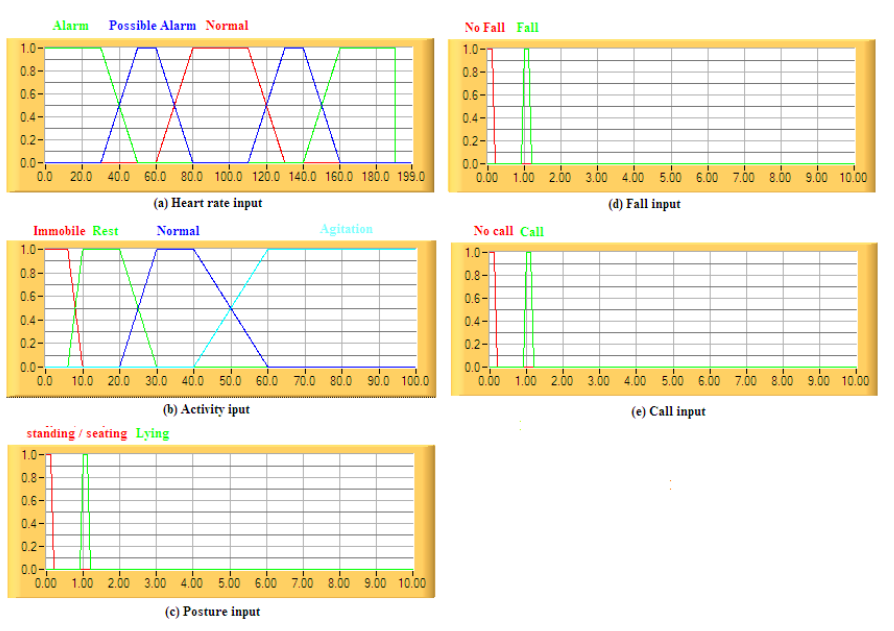

Fig. 5. Fuzzy sets defined for Vital data inputs.

Four membership functions are set up in this numerical scale according to the following fuzzy levels: no signal, normal, possible alarm and alarm as it is shown in figure 4. The second input is associated with the SNR (Signal-to-noise ratio) calculated on each microphone (two microphones are used in the current application), and this input is split into three fuzzy levels: low, medium and high.

RFpat provides physiological data to EMUTEM platform. RFpat produce five inputs:

- Heart rate is fuzzified with three fuzzy levels: normal, possible alarm and alarm.

- Activity has four fuzzy sets: immobile, rest, normal and agitation. The trapezoid function displays all these linguistic variables.

- Posture is represented by two membership functions standing up / seating down and lying.

- Fall and call have also two fuzzy levels: Fall/Call and No Fall/Call and a singleton function is associated to these linguistic variable.

Parameters of membership functions associated to the heart rate and the activity, are adjustable according to the monitored elderly person. An automatic procedure to adapt these intervals based on 30 minutes recording was proposed.

For each infrared sensor a counter of motion detection with three fuzzy levels (low, medium, high) is associated. It is reset every 5 seconds. A global counter for all infrared sensors with three fuzzy membership functions (low, medium, high) is also used and is reset every 60 seconds. A trapezoid membership function is chosen to characterize these fuzzy sets.

A singleton membership function is assigned to each change state sensor with two linguistic variables: On and Off.

The last input which is the time. It has two membership functions: day and night. These two membership functions are also adaptable to each patient habits. Trapezoid functions are used to divide the time input.

In order to reach the objective of the EMUTEM platform which is the identification of distress situations of an elderly 
person at home, two outputs are associated to the fuzzy inference engine of EMUTEM platform.

The first one is called Alarm with two linguistic variables normal and alarm.

To refine the decision of the EMUTEM platform a second output is added to its fuzzy inference system component. This second output is named Localization. It is a very important information for the diagnostic. The identification of the position of the monitored person during a distress situation is helpful knowledge for medical diagnosis.

Two membership function models are selected: Gaussian functions are chosen for the alarm outputs; Trapezoid functions for the localization output where the classical areas of a house are its fuzzy levels or linguistic variables.

\section{Fuzzy Rules Aggregation and Defuzzification}

The EMUTEM fuzzy inference engine is formulated by two groups of fuzzy IF-THEN rules. One group controls the output variable localization according to values of the input variables issued from infrared sensors and the SNR of each microphone. The other group controls the output linguistic variable alarm according to all inputs. These fuzzy rules are decided through experimentation and according to some expert knowledge.

A confidence factor is assigned for each rule. Outputs used in a rule are multiplied by the confidential factor issued from each subsystem. Thus the rule's output value depends on the reliability of each subsystem and the sureness of rules. To aggregate these rules we have chosen the Mamdani model instead of the Takagi Sugeno. These two models are available under the EMUTEM fuzzy logic component. Mamdani model offers us a good way of modeling the normal and distress situations, because these two classes don't form a clean partition but a fuzzy one.

After rules aggregation the defuzzification is performed by the smallest value of maximum method for the alarm output in order to obtain also a confidence level of each alarm's decision, and the centroid of area for the localization output.

Each subsystem specifies the situation of the elderly person and the degree of anxiety. When a decision is very complicated, that is, there are many decisions; the fuzzy method is especially useful. It is also easy to check, modify, and add/delete every fuzzy variable for better automated analysis. To summarize the proposed data fusion method, the proposed fuzzy destress situation classifier is comprised of two major function blocks, decisions of each subsystems and fuzzy classifier as shown in Figure 3. Decisions of subsystems are utilized as inputs for the classification using the fuzzy classifier. The derived parameters will be exported to the latter for the classification. The Mamdani fuzzy method is used. Fuzzy logic if-then rules are formed by applying fuzzy operations to these membership functions for given inputs and outputs. The resulting output membership functions are added together using desired weights yielding a sort of probability function. The resulting output areas can then be used to estimate the expected value of the output variable by using the specified defuzzification method. In general, any fuzzy classifier has to undergo iterative adjustment in terms of fuzzy variables, including the choice of membership functions, and the definition of rules in the knowledge base.

\section{Software Architecture}

Figure 6 provides a synoptic block-diagram scheme of the software architecture of the EMUTEM system; it is implemented under Labwindows CVI and C++ software. It is developed in a form of a design component.

We can distinguish three main components, the acquisition module, the synchronization module and the fuzzy inference component. It can run off-line by reading data from a data base or online by processing in real time data acquired via the acquisition module.

To avoid the loss of data, a real time module with two multithreading tasks is integrated in the synchronization component. The EMUTEM system is now synchronized on the smart home sensors (Gardien) subsystem because it has the lowest sampling rate $(2 \mathrm{~Hz})$ and periodicity. The data from others modalities are memorized and used several time in order to have the same sampling rate (RFPAT data is used 60 times). This multithreading technique allows a maximum delay for an asynchronous data (Sound or alarm from RFPAT) of $0.5 \mathrm{~s}$.

We have developed Fuzzy tools for the data fusion step. These tools allow the easy configuration of input/output intervals of Fuzzification, the writing of fuzzy rules and the configuration of the defuzzification method. It is also possible to add others modalities to this fuzzy inference system which make the EMUTEM platform flexible. Two outputs are associated to the fuzzy inference system, Alarm for distress situation detection and Localization for elderly person position detection.

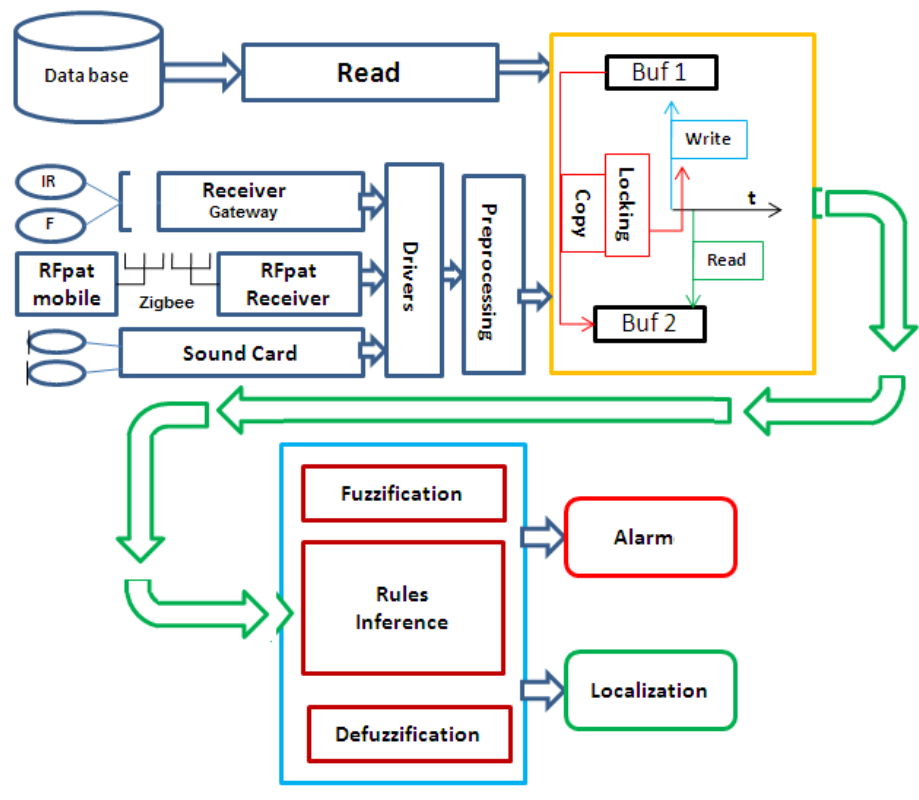

Fig. 6. EMUTEM software components design . 


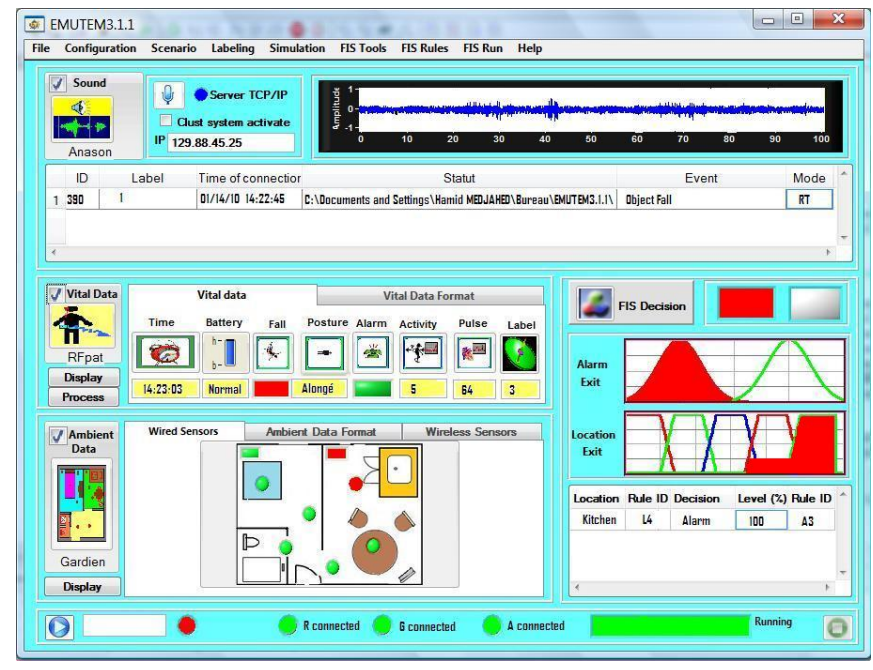

Fig. 7. Graphical Interfaces for Intelligibility.

\section{E. Graphical Interfaces for Intelligibility}

To offer enhanced intelligibility for the EMUTEM platform, a graphical user interface (GUI) was developed for this task. It facilitates the various configurations required for the functioning of the platform. It is very useful for users.

Figure 7 shows this general graphical interface. It is possible to build up membership functions of inputs and outputs and displaying them under this graphical interface.

We could also write rules via this graphical interface. It is also possible to write rules to text file by using a specific language, that we have developed, understandable by our system.

These Graphical interfaces provide EMUTEM with a useful simplicity for users and with a flexibility that allows adding other modalities. They allow experts to add their knowledge in a user friendly way.

\section{The In Home Monitoring Database}

In order to test and to validate the EMUTEM platform, an environment for acquiring and recording a multimodal database (HOMECAD [7]) is integrated under this platform. To record this multimodal database, users can interpret elderly activities by following a reference scenario which summarizes the everyday life of elderly persons. These scenarios are divided into two categories: either a critical scenario with one or more distress events, or a normal one without any distress event. To define these scenarios a study is performed and they were instigated under CompanionAble European project. In this project, some elderly living alone were followed up by a co-worker team, in order to summarize and to describe their daily routine. The recorded multimodal database gathers physiological data, environment sounds and others different information gathered by ubiquitous sensors associated to Gardien subsystem.

First recordings were performed in our laboratory. Participants in this study were 10 adult volunteers who were recruited from the university and from the community. Participants ranged in age from 25 to 50 years. Participants were asked to perform several activities according to the used reference scenario. As each scenario lasts 10 minutes, this task corresponds to 200 minutes of recorded data.

The second stage of recording occurred in Broca Hospital in Paris under the framework of CompanionAble project. Ten adult volunteers from the community were enrolled to participate in this recording stage. The participants profile varied, ranged in age from 65 to 75 years, and the sample was $60 \%$ female and $40 \%$ male. The last stage of recording was also performed under the CompanionAble project and was performed in SmartHomes at Eindhoven. Fifteen adult volunteers were involved in this recording. For both stages of recording, a scenario lasts 15 minutes and each participant performs three different scenarios.

An additional process of simulation is also integrated in our platform as a way to overcome the lack of experimental data and the difficulty of recording some medical data such as the cardiac frequency during distress situations.

Taking into account the multimodality character of the data, a multidimensional indexing process is used in order to obtain a full description of data sets. In order to index our multimodal database, we have retained the SAM standard indexing file [23] generally used for speech database description. It indicates information about the file and describes it by delimiting the useful part for further analyzing and processing.

\section{EXPERIMENTAL RESUlTS AND VALIDATION}

The realization of an experimental process requires the use of appropriate metrics for evaluating the performance of the platform by comparing the system's results to expected results. It is useful to describe some parameters or metrics that allows an objective evaluation of the results. To perform this experimental process we have selected the following metrics:

- Sensitivity (Se): Identify patterns of real abnormal situations as distress ones.

- Specificity (Sp): Don't identify normal situations as distress situations.

- Error rate (Err): It is the ratio between the number of the misclassified samples and the total number of the samples.

- Perfect classification $(P c)$ : It is the ratio between the number of the correct classified samples and the total number of the samples.

Indices of sensitivity (Se), specificity (Sp), error rate (Err) and perfect classification $(\mathrm{Pc})$ are calculated from rates of true /false positive/negative, marked respectively with these symbols TP, FP, TN, and FN.

$$
\begin{gathered}
S e(\%)=\frac{T P}{T P+F N} \times 100 \\
S p(\%)=\frac{T N}{T N+F P} \times 100 \\
\operatorname{Err}(\%)=\frac{F N+F P}{T N+F N+F P+T P} \times 100 \\
P c(\%)=\frac{T N+T P}{T N+F N+F P+T P} \times 100
\end{gathered}
$$




\begin{tabular}{|c|c|r|r|}
\cline { 3 - 4 } \multicolumn{2}{c|}{} & \multicolumn{2}{c|}{ EMUTEM Classification } \\
\cline { 3 - 4 } \multicolumn{2}{c|}{} & Distress sequence & Normal sequence \\
\hline \multirow{2}{*}{$\begin{array}{l}\text { Simulated } \\
\text { sequences }\end{array}$} & Distress sequence & 68 & 2 \\
\cline { 2 - 4 } & Normal sequence & 1 & 29 \\
\hline
\end{tabular}

TABLE I

CLASSIFICATION RESULTS FOR ALARM OUTPUT WITH SIMULATED DATA BY USING 10 RULES

The just exposed metrics of the statistic data are very important to estimate the classification accuracy.

In order to demonstrate the effectiveness of this software, firstly we started by using simulated data in order to validate each rule. This first step of simulation gave very promising results for the alarm generation and localization without any false decision for each rule.

After that 100 sequences of simulation are used to test EMUTEM, where 70 sequences represent distress situation and 30 sequences represente normal situation.

In order to evaluate the classification accuracy the confusion matrix has been calculated for this simulation. Table I displays the obtained results with 10 rules.

\begin{tabular}{|l|c|}
\hline Sensitivity Se & $97 \%$ \\
\hline Specificity Sp & $96 \%$ \\
\hline Error rate Err & $3 \%$ \\
\hline Perfect classification Pc & $97 \%$ \\
\hline
\end{tabular}

TABLE II

PERFoRmANCE INDICES FOR ALARM OUTPUT OBTAINED WITH SIMULATED DATA BY USING 10 RULES

From table I we can deduce some indices of performance which are displayed in table II. The obtained results of EMUTEM's performance are good and they demonstrate the reliability of the EMUTEM platform.

Even if we have $3 \%$ of misclassified sequences, this error rate could be overcome by adding to the fuzzy inference system the right rules that take into account the misclassified situations, and also by associating to each rules the right weight.

For the localization output, also we have obtained about $98 \%$ of good localization.

Figure 8 shows results for a steam of data and summarizes the process of the fuzzy inference system. The first step of this process is to determine the output of each membership function assigned to each input. Then the outputs of the rules are calculated by applying the fuzzy operators (AND and OR) in the antecedent part of all rules, using the T-norm and Tconform operators respectively and the implication from the antecedent to the consequent, using T-norm operator. After that the aggregation of the consequents across the rules of each output is performed by using the Mamdani model, it is the unification process of the all rule outputs. Finally the process of defuzzification is done by extracting out one crisp value as the output, the smallest value of maximum method is chosen for the alarm output and the centroid of area for the localization

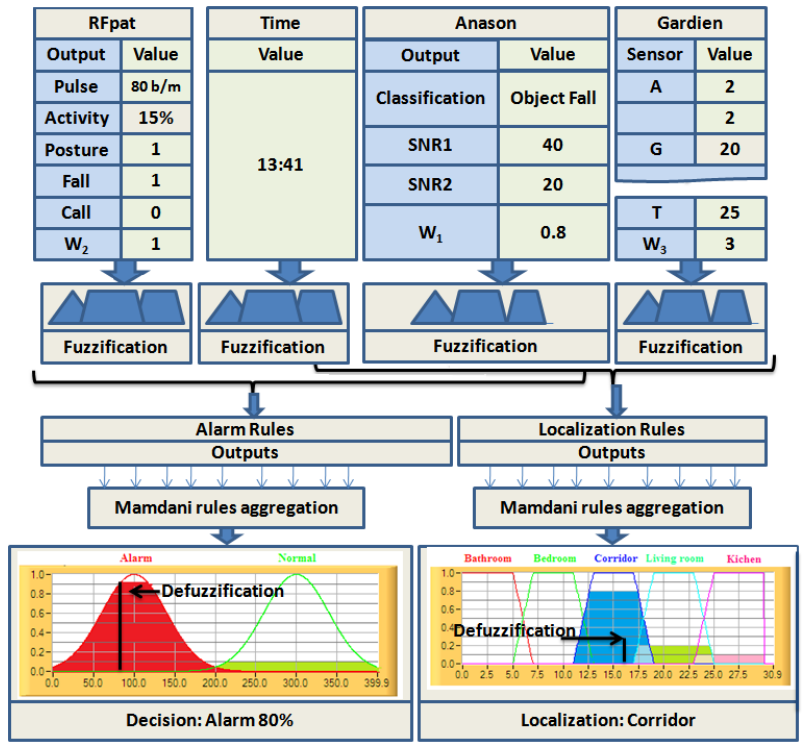

Fig. 8. Results for a stream of data

output.

The EMUTEM platform was tested with the HOMECAD database described in the previous section. This first study is devoted to the evaluation of the system by taking into account rules used in this fuzzy inference system. The strategy consisted in realizing several tests with different combination rules, and based on the obtained results, one rule is added to the selected set of rules, or removed from this selected set of rules in order to get the missed detection. Based on the obtained results some weights of rules are also changed. With this strategy good results are reached for the alarm output with 10 rules and 16 rules for the localization outputs. The data fusion classification using the fuzzy logic approach has given better performance results when compared to analytical method and using artificial neural networks (ANN) for classification. More over problems associated with conventional neural network architectures such as learning rate limitation and difficulty in selecting the optimal number of hidden units are eliminated. Finally the accuracy of the classifier is shown in table III.

\begin{tabular}{|l|c|r|}
\cline { 2 - 3 } \multicolumn{1}{c|}{} & Fuzzy classifier (10 rules) & ANN (3 hidden layers) \\
\hline Se & $95 \%$ & $87 \%$ \\
\hline Sp & $93 \%$ & $85 \%$ \\
\hline Err & $5 \%$ & $12 \%$ \\
\hline Pc & $95 \%$ & $88 \%$ \\
\hline
\end{tabular}

TABLE III

PERFORMANCE INDICES FOR ALARM OUTPUT OBTAINED WITH FUZZY CLASSIFIER AND ANN METHOD

With this strategy good results are reached for the alarm output with 10 rules and 16 rules for the localization outputs, about $95 \%$ of good Alarm detection and $97 \%$ for good Localization. The rate of misclassification for the alarm output corresponds to situation that are not detectible by the sensors used by EMUTEM and also the difficulty to find the right 
rule to overcome these situations. For the localization output the error rate could be justified by the fact that we use an area where an apartment is simulated thus the calibration of infrared sensors is very hard.

These first results encourage us to perform further tests in real time in order to have an effective evaluation of the EMUTEM platform.

\section{CONCLUSION}

In this work, we focused on the area of automatic home healthcare telemonitoring, in which health information is automatically collected with the help of sensors and processed by special algorithms and fused in order to make good decisions about elderly persons living alone at home. The proposed multimodal data fusion method based on fuzzy logic represents a fast and easy tool for the interpretation of the fuzzy decision process even for very high dimensional input spaces and allows fast detection of errors. The impact of the input features plays an important role on the final decision process. The algorithm supports the implementation of the expert's knowledge and optimized the system easily. It is possible to increase the performance further by adding more related input variables and with more data to enrich the knowledge in the rules.

The EMUTEM platform which encloses this architecture is implemented and validated by simulation and by using real data. The experimental results were accurate and robust. The main advantage of the presented method consists of the lowcomputational expenses inherited from the characteristics of fuzzy systems. This approach allows the easiest combination between data and adding other sensors.

The fuzzy logic decision module reinforces the secure detection of older person distress events and his localization. This constitutes a great asset for the EMUTEM system, by offering the possibility in the near future, to implement a very intelligent remote monitoring system in care receiver houses, thus to build very reliable smart houses.

\section{ACKNOWLEDGMENT}

The authors would like to thank the contribution of European Community's Seventh Framework Programme (FP7/2007-2011), CompanionAble Project (grant agreement n.216487).

\section{REFERENCES}

[1] H. Bouma, Gerontechnology: A framework on technology and aging, In H. Bouma and J. Graafmans, editors, Gerontechnology, ISO Press. pages 1-6, Amsterdam, 1993.

[2] D. Doermann and D. Mihalcik, A system approach to achieving carernet, an integrated and intelligent telecare system, IEEE Trans Biomed Eng, 2:1-9, 1998.

[3] N. Noury, P. Barralon, G. Virone, P. Boissy, M. Hamel, and P. Rumeau, A smart sensor based on rules and its evaluation in daily routines, in Proc of the IEEE-EMBC, pages 3286-3289, Cancun, Mexico, september 2003.

[4] G. Elger and B. Furugren, "smartbo", an ICT an computer-based demonstration home for disabled people, in Proc. of the 3rd TIDE Congress : Technology for Inclusive Design and Equality Improving the Quality of Life for the European Citizen, Helsinki, Finland, 1998.
[5] G.A.W. West, S. Greenhill and S. Venkatesh, A probabilistic approach to the anxious home for activity monitoring, in Proc. 29th Annual International Computer Software and Applications Conference: COMPSAC, pages 335-340, Edinburgh, Scotland 2005.

[6] J.E. Rougui, D. Istrate, W. Souidene, Audio Sound Event Identification for distress situations and context awareness, EMBC2009, September 2-6, Minneapolis, USA, 2009, pp. 3501-3504.

[7] H. Medjahed, D. Istrate, J. Boudy, F. Steenkeste, J.L. Baldinger, I. Belfeki, V. Martins and B. Dorizzi, A Multimodal Platform for Database Recording and Elderly People Monitoring, BIOSIGNALS 2008, 28-31 janvier 2008, Funchal-Madeira, Portugal, pp.385-392.

[8] R. Cowell, A. Dawid, S. Lauritzen, and D. Spiegelhalter, Probabilistic Networks and Expert Systems, 1999, ISBN : 0-387-98767-3.

[9] G. Dreyfus, J.M Martinez, M. Samuelides, M. Gordon, F. Badran, $\mathrm{S}$. Thiria and L. Hrault, Réseaux de neurones. Méthodologie et applications, Eyrolles, 2002.

[10] C.J.C. Burges, A tutorial on SVM for Pattern Recognition. Data Mining and Knowledge Discovery, volume 2, 1998, pp. 121167.

[11] D.A. Reynolds, Speaker identification and verification using gaussian mixture speaker models. Speech Comm, 17:91108, January 1995.

[12] BitCloud User Guide, Atmel, 8199-MCU Wireless- 02/09

[13] S. Stillman and I. Essa. Toward reliable multimodal sensing in aware environments, Perceptual User Interfaces (PUI 2001) Workshop (held in conjunction with ACM UIST 2001 Conference), Orlando, Florida, November 15-16, 2001.

[14] Lawrence A. Klein. Fuzzy set theory in medical diagnosis, IEEE Tr. On Syst.,Man, and Cybernetics, pages 260-265, March/April 1986.

[15] S.J Russell and P. Norvig, Probabilistic reasoning in artificial intelligence: a modern approach, Upper Saddle River 2nd, pages 492-494, N.J Prentice Hall, 2003.

[16] G. Shafer, A mathematical theory of evidence. Princeton University Press, Princeton, NJ, 1976.

[17] C. Bishop. Neural networks for pattern recognition, Oxford, 1995.

[18] C.J.C. Burges. A tutorial on SVM for pattern recognition, Data Mining and Knowledge Discovery, Volume 2, pages 121-167, 1998.

[19] L. Zadeh, Fuzzy sets. in Fuzzy Models for Pattern Recognition: Methods That Search for Structures in Data, edited by J. Bezdek and S. Pal, IEEE Press, pages 35-45, New York 1992.

[20] W. Pedrycz, Fuzzy sets in pattern recognition: methodology and methods. Pattern Recognition, 23(1/2):121-146, 1990.

[21] R. Cowell, A. Dawid, S. Lauritzen, and D. Spiegelhalter, Probabilistic Networks and Expert Systems, 1999, ISBN : 0-387-98767-3.

[22] J.S.R. Jang, C.T Sun and E. Mizutani, Neuro-Fuzzy and Soft Computing : A Computational Approach to Learning and Machine Intelligence, Prentice Hall Upper Saddle River, NJ 1997.

[23] D. Well, J. Barry, W. Grice, M. Fourcin, , and A. Gibbon., Esprit project 2589-multilingual speech input/output assessmen, methodology and standardization, In final report, Technical Report SAM-UCLG004, University College London, 2004. 